\title{
Video instruction without additional voice feedback exercises is insufficient for initial acquisition of basic life support skills in a self-learning station.
}

\section{Mpotos $\mathbf{N}^{1}$, Calle PA ${ }^{1}$, Peersman $\mathbf{W}^{2}$, Valcke $\mathrm{MA}^{3}$, Monsieurs KG ${ }^{1}$}

1Emergency Department, Ghent University Hospital, De Pintelaan 185, B-9000 Ghent, Belgium

${ }^{2}$ Department of General Practice and Primary Health Care, Ghent University, De Pintelaan 185, B-9000 Ghent, Belgium

${ }^{3}$ Department of Educational Studies, Ghent University, H. Dunantlaan 2, B-9000 Ghent, Belgium

\section{Introduction}

European Resuscitation Council 2010 Guidelines encourage short video/computer self-learning (SL) courses, with minimal or no instructor coaching. We analysed the learning efficacy of an abbreviated Mini Anne ${ }^{\mathrm{TM}}$ (Laerdal, Norway) video followed by refinement with voice feedback exercises on initial acquisition of Basic Life Support (BLS) skills in a SL station.

\section{Methods}

One hundred and twenty-five pharmacy students (86\% without previous training), were trained in a SL station on a manikin using learning-while-watching video instructions followed by exercises with concurrent voice feedback (Resusci Anne Skills Station ${ }^{T M}$ Laerdal, Norway). The proportions of students with adequate BLS skills (mean compression depth $\geq 50 \mathrm{~mm}$, mean compression rate $100-120 / \mathrm{min}$, complete release $<5 \mathrm{~mm}$ and mean ventilation volume $400-1000 \mathrm{ml}$ ) were measured at baseline, after video training and after subsequent training with voice feedback.

Fig. 1: Participants flow chart

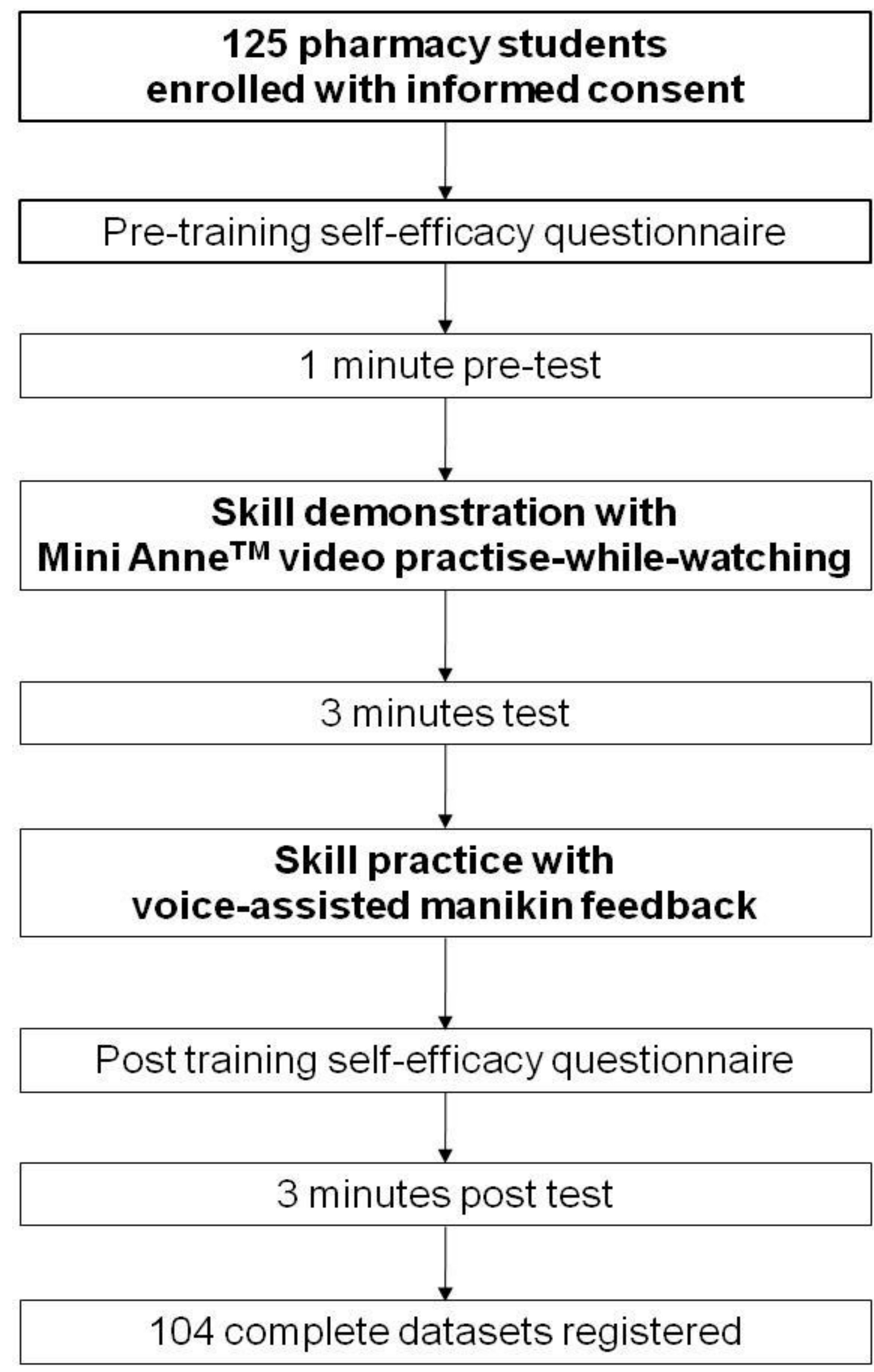

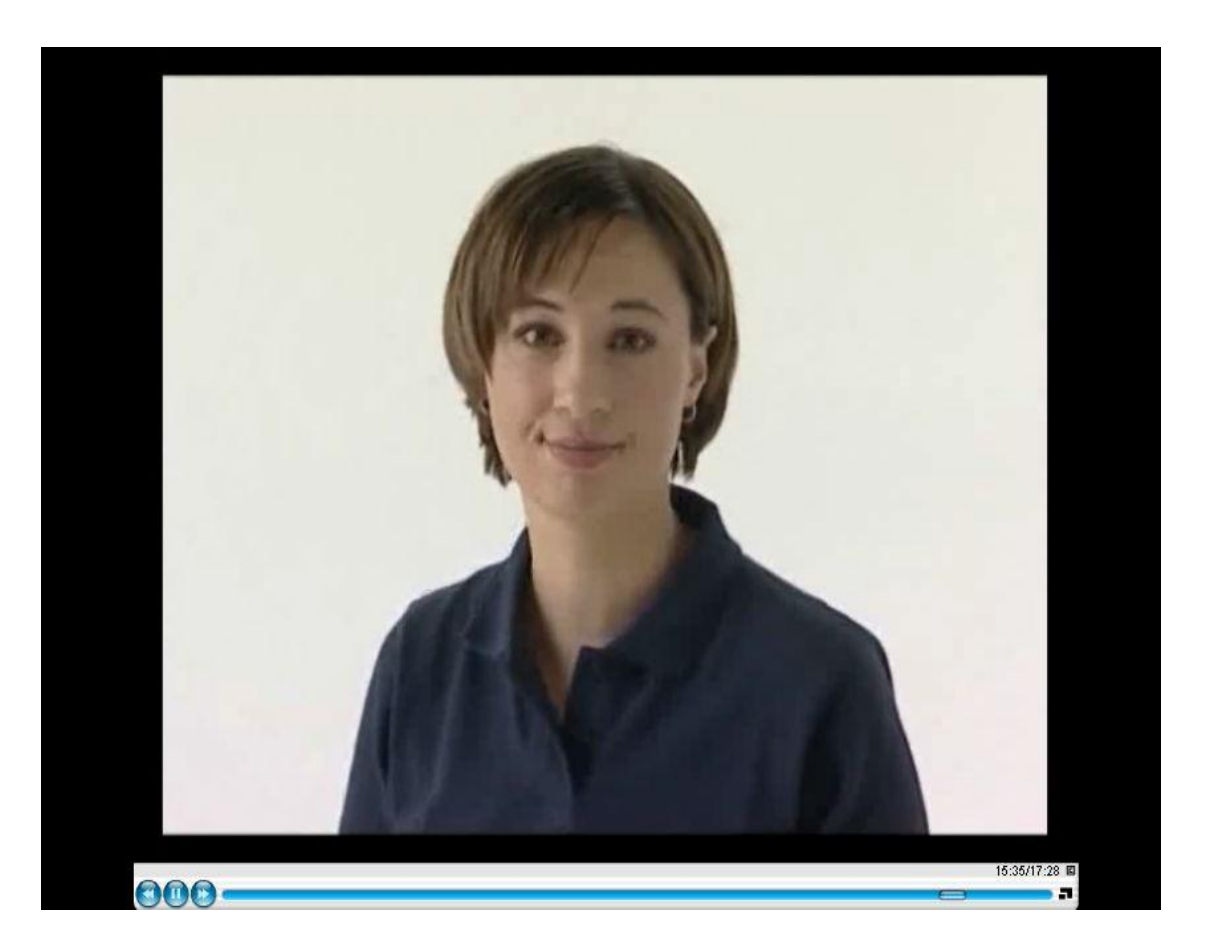
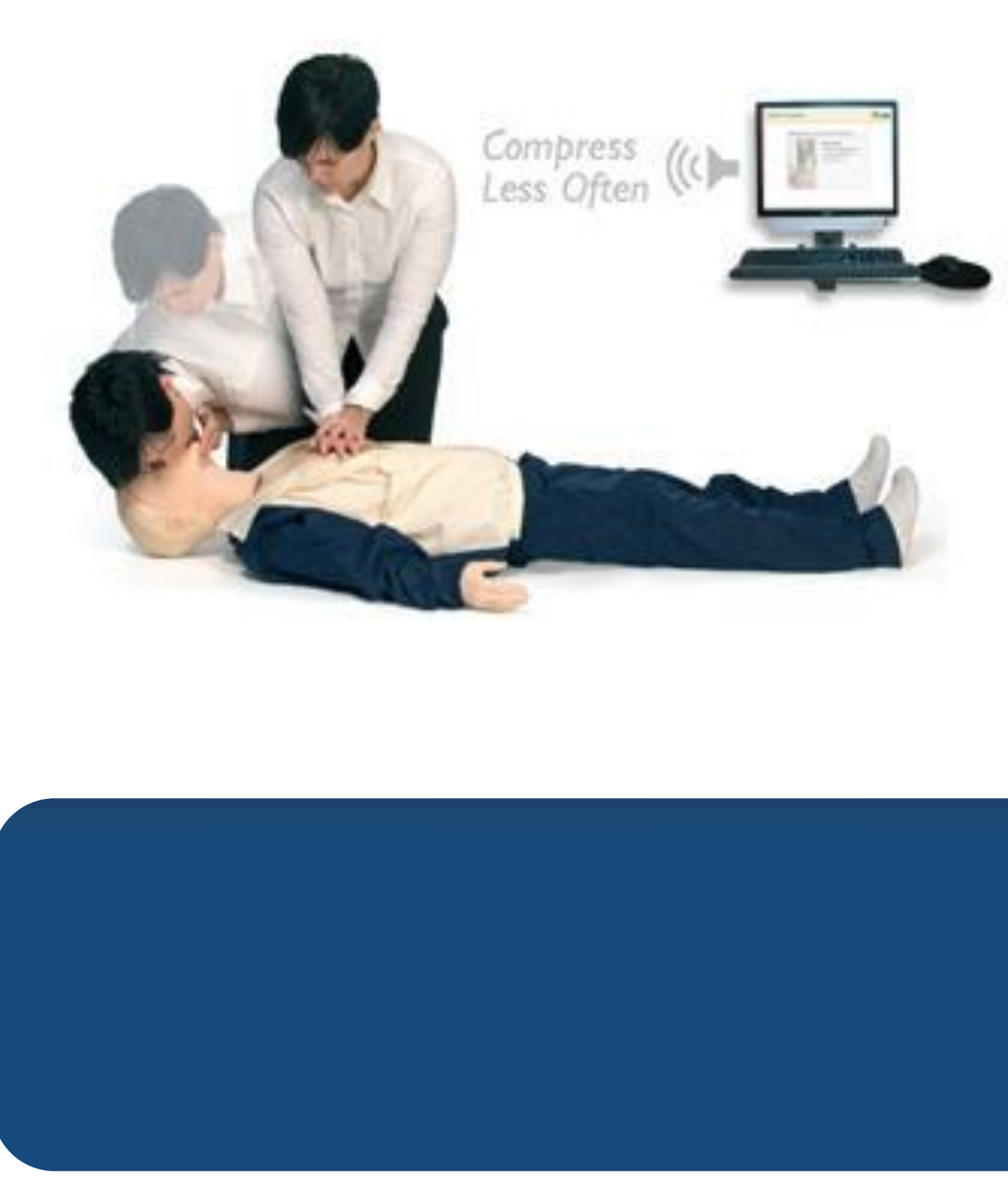

Conclusion

To acquire BLS skills, video training alone was not effective and only improved compression rate and ventilation skills in some of the students. Additional voice feedback exercises resulted in skills mastery in the majority of students.

kritieke diensten

$\sqrt{2}$ Universitair Ziekenhuis Gent

Contact: Nicolas.Mpotos@UGent.be 